\title{
Note de correction: Machine biaxiale sur la ligne de lumière Diffabs pour l'étude des propriétés mécaniques de films minces déposés sur substrats polymères
}

Correction note: Biaxial machine at Diffabs beamline for studying mechanical properties of thin films deposited onto polymer substrates

Matériaux \& Techniques 103, 610 (2015), https://doi.org/10.1051/mattech/2015057

Damien Faurie ${ }^{1, *}$, Soundes Djaziri ${ }^{2,3}$, Pierre-Olivier Renault ${ }^{3}$, Eric Le Bourhis ${ }^{3}$, Philippe Goudeau ${ }^{3}$, Guillaume Geandier ${ }^{4}$, and Dominique Thiaudière ${ }^{5}$

${ }^{1}$ LSPM-CNRS, Université Paris 13, Université Sorbonne Paris Cité, Villetaneuse, France

2 Max-Planck-Institut fur Eisenforschung GmbH, Max-Planck-Straße 1, 40237 Düsseldorf, Germany

${ }^{3}$ Institut Pprime (UPR 3346 CNRS), Université de Poitiers, Bd Pierre et Marie Curie, 86962 Futuroscope Cedex, France

${ }^{4}$ Institut Jean Lamour (UMR 3079 CNRS), Université de Lorraine, Parc de Saurupt, CS 50840, 54011 Nancy Cedex, France

${ }^{5}$ Synchrotron SOLEIL, L'Orme des Merisiers, Saint-Aubin, BP 48, 91192 Gif-sur-Yvette Cedex, France

Reçu le 31 juillet 2018 / Accepté le 31 juillet 2018

Une erreur s'est produite dans l'article «Machine biaxiale sur la ligne de lumière Diffabs pour l'étude des propriétés mécaniques de films minces déposés sur substrats polymères » publié dans le volume 103, 610 (2015). L'affiliation correcte des auteurs Pierre-Olivier Renault, Eric Le Bourhis et Philippe Goudeau est «Institut Pprime (UPR 3346 CNRS), Université de Poitiers, Bd Pierre et Marie Curie, 86962 Futuroscope Cedex, France ». L'éditeur s'excuse pour cette erreur.

\footnotetext{
* e-mail: faurie@univ-paris13.fr
} 